Chrischta Ganz

\section{Heilpraktikerschule, Luzern}

Die HPS Luzern befindet sich zurzeit noch in der Stadt Luzern, wird aber im November dieses Jahres nach Ebikon LU in neue Schulräumlichkeiten ziehen, da der alte Standort zu klein geworden ist. Der erste Spatenstich wurde am 14. Januar 2014 gefeiert - vom Regen liessen sich die Anwesenden nicht stören. Die Vorfreude auf «mehr Platz» (was acht Schulräume, drei Geschosse, 1250 Quadratmeter und ein Atrium bedeutet) war grösser.

Die HPS Luzern wird geleitet von den TCM- und Shiatsu-Therapeuten Ulrike von Blarer Zalokar (Gründerin) und Peter von Blarer sowie dem Betriebswirt Hein Zalokar (Abb. 1). Ulrike von Blarer Zalokar und Peter von Blarer sind Gründungsmitglieder

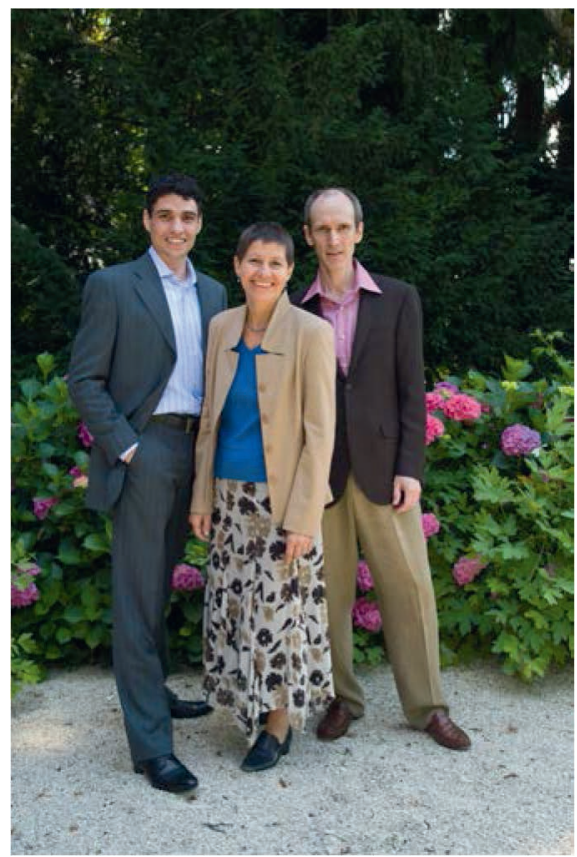

Abb. 1. Die Schulleitung der HPS Luzern: Hein Zalokar, Ulrike von Blarer Zalokar und Peter von Blarer.

\section{Fortbildung}

Schweiz Z Ganzheitsmed 2014;26:162-164 DOI: 10.1159/000362899

Online publiziert: Mai 5, 2014

\title{
Naturheilpraktikerschulen der Schweiz - Teil 2
}

In der zweiten Folge von «Naturheilpraktikerschulen der Schweiz» werden die Heilpraktikerschule (HPS), Luzern, und die Akademie für Naturheilkunde (ANHK), Basel, sowie das SAKE Bildungszentrum, Bern, vorgestellt.

der SBO-TCM (Schweizerische Berufsorganisation für Traditionelle Chinesische Medizin). Ulrike von Blarer Zalokar ist den Lesenden vom Expertengespräch zum Thema Ernährung in Ausgabe 6/13 der SCHWEIZERISCHEN ZEITSCHRIFT FÜR GANZHEITSMEDIZIN bekannt.

Die HPS Luzern startete 1985 als Shiatsu-Schule. Bald kamen die Traditionelle Europäische Naturheilkunde (TEN) und die Traditionelle Chinesische Medizin (TCM) dazu. Ihre West-TCM-Variante (westliche Heilkräuter und Nahrungsmittel werden gemäss Prinzipien der TCM eingesetzt) ist Pionierarbeit. Die erst intern publizierten Fachbücher sind seit 2009, die Kochbücher seit 2004 offiziell verlegt. 2013 wurde das TCMAngebot der HPS mit Tuina und der Chinesischen Arzneimittellehre vervollständigt, sodass heute alle TCMMethoden (Akupunktur, Chinesische Arzneimittel, Diätetik West-TCM, medizinisches QiGong, Phytotherapie West-TCM, Tuina) an der HPS zu lernen sind. Mit dem neuen Berufsbild "Alternativmedizin» (AM) wird die TEN-Ausbildung der HPS Luzern nun auf die Humoralmedizin als Hauptmedizinalsystem ausgerichtet.

Seit 2003 ist auch Hein Zalokar, Sohn von Ulrike von Blarer Zalokar, in der Schulleitung engagiert, und mit ihm sind weitere Ausbildungsgänge diverser Komplementärtherapien dazugekommen. Die Schule ist seit 2004 EduQua-zertifiziert. Dass die familiäre Atmosphäre trotz der Grösse hat bestehen können, zeichnet die
Schule ebenso aus wie die 29-jährige Konstanz in der Schulleitung. Die HPS Luzern bietet Kurzausbildungen wie auch Studiengänge in den Fachrichtungen der Alternativmedizin TCM, TEN und Ayurveda (ab Mitte 2015) sowie spezifische Vorbereitungskurse für die eidgenössische Heilpraktikerprüfung an.

Der Schule angeschlossen ist ein Ambulatorium mit fünf Praxisräumen (unter anderem Praktikumsplätze für Studierende). Zusätzlich bestehen Praktikumspartner im vietnamesischen Hanoi und im chinesischen Kunming.

\section{Naturheilkunde - \\ Naturheilpraktiker/innen}

Die Naturheilkunde wird an der HPS Luzern als ein grosses Feld von Heilungsmethoden gesehen, die innerhalb ihres Medizinalkonzepts (TCM, TEN oder Ayurveda) die Gesundung eines Kranken unterstützen oder zur Vorbeugung beitragen. Wichtig ist für die Schulleitung, dass Naturheilkunde in einer schulmedizinischen Kultur ausgeübt und dass dies als Ergänzung in gegenseitiger Bereicherung unterrichtet und gelebt wird.

Wer den Beruf des Naturheilpraktikers erlernen will, soll nach Meinung des HPS-Teams neugierig sein, eine Berufung verspüren, ehrlich und eigenständig sein sowie Menschen gern haben. Vernetztes Denken und prozessorientiertes Arbeiten, analytische Fähigkeiten sowie Empathie gehören ebenfalls dazu - und Respekt vor den

\section{KARGER}

Fax +497614520714 Information@Karger.com www.karger.com
(๑) 2014 S. Karger GmbH, Freiburg
Chrischta Ganz

Praxis für Naturheilkunde

Friedhofstrasse 15, 8636 Wald ZH, Schweiz

naturheilpraxis@chrischtaganz.ch www.chrischtaganz.ch 


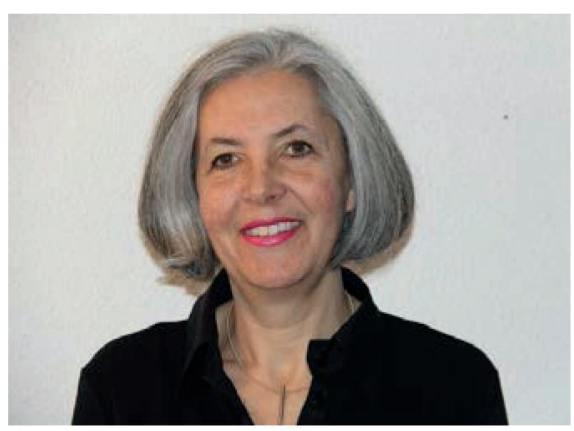

Abb. 2. Sabine Richner Rossi, Institutsleitung der ANHK, Basel.

Grenzen sowohl der Naturheilkunde als auch der Schulmedizin.

Die Tatsache, dass der Frauenanteil im Bereich der Naturheilkunde so hoch ist, wird auf die gesellschaftliche Struktur zurückgeführt: Frauen arbeiten öfter Teilzeit als Männer, da sich Männer häufig in der Ernährerrolle sehen. Ebenso gründet das Interesse der Frauen oft auf ihren Erfahrungen mit kranken Kindern oder bei Fragen zur Ernährung.

\section{Persönlichkeitsentwicklung}

Seit 2007 werden auch humanistische Grundsätze der therapeutischen Tätigkeit gelehrt und geübt und bringen die Therapeuten und Therapeutinnen persönlich weiter. Dies ist sehr wichtig, da die Patienten in der heutigen Naturheilpraxis immer mehr Entschleunigung suchen und der Beruf herausfordernd ist, da «man gut sein muss, um sich durchzusetzen, auch bezüglich Marketing und Kommunikation», so die Meinung der Schulleitung der HPS Luzern.

\section{Akademie für Naturheilkunde, Basel, und SAKE Bildungszentrum, Bern}

Die ANHK in Basel wird von Sabine Richner Rossi (Institutsleitung) (Abb. 2) und Sina Maier-Faller (Administration und Beratung) geleitet.

Die Schule gehört zur Basler Bildungsgruppe (BBG), einem Verbund von renommierten Privatschulen in der Schweiz und in Deutschland. In der ANHK werden die Ausbildungsgänge Dipl. Naturarzt Akupunktur TCM, Naturarzt TEN, Naturarzt Homöopathie ANHK, Dipl. Homöopath ANHK sowie Dipl. Akupunkteur ANHK angeboten.

Innerhalb der Schule gibt es Fachvorstände für jede Fachrichtung (TCM, TEN, HOM) sowie für die Schulmedizin. Die EduQua-zertifizierte Schule wurde 1994 durch engagierte Ärzte und Heilpraktiker gegründet und 1997 als selbstständiges Institut in die BBG integriert. Die ANHK wird ihrem Anspruch eines hohen Ausbildungsniveaus in der Schulmedizin und der optimalen Vorbereitung auf die kantonale Prüfung Basel gerecht.

Das SAKE Bildungszentrum Bern wurde 1986 als Schule für asiatische Körper- und Energiearbeit in Bern mit dem Ziel gegründet, östliche und westliche Naturheilkunde in die von der Schulmedizin geprägte Welt $\mathrm{zu}$ integrieren. Geleitet wird das SAKE Bildungszentrum, das seit 2005 EduQua-zertifiziert und Mitglied der Konferenz Höherer Fachschulen Alternativmedizin (hfam) sowie des Verbands Schweizerischer Privatschulen (VSP) ist, von Lotti Westmoreland (Schulleitung) und Martin Loeffel (Leitung Administration). Im Jahr 2007 wurden die Ausbildungen der GAM-Fachschule Bern integriert. Angeboten werden Diplomausbildungen in der TCM (Akupunktur, Tuina, Chinesische Heilkräuter) und in der TEN sowie verschiedene Komplementärtherapien.

In der Ausbildung der Fächer der TEN (Abb. 3) kooperiert die ANHK Basel mit SAKE Bern; beide Schulen führen die Ausbildung weiterhin nach ihren Vorgaben und in eigener Verantwortung durch. In einer ersten Phase werden einzelne Module am Standort der Partnerschulen durchgeführt. Mit dieser Massnahme können die beiden Institute den stetig wachsenden Anforderungen im Bildungswesen gerecht werden und Synergien nutzen.

\section{Naturheilkunde - \\ Naturheilpraktiker/innen}

Nach Meinung der ANHK/SAKE sind für den Beruf des Naturheilpraktikers Menschen geeignet, die Belastbarkeit, hohe Sozialkompetenz, Verantwortungsbewusstsein, gesunden Menschenverstand, emotionale Balance, Offenheit für andere Menschen und deren psychosoziales Umfeld sowie Selbstkenntnis und Auseinandersetzung mit der Rolle als Therapeut/in in sich tragen oder diese $\mathrm{zu}$ entwickeln vermögen.

Für Sabine Richner Rossi ist die Naturheilkunde sowohl Alternative als auch Ergänzung zur Schulmedizin. Auf die Frage, warum so viel mehr Frauen vertreten sind, meint sie:

«Das betrifft ja nicht nur die momentanen Lehrgänge - es war/ ist seit Jahrzehnten so. Frauen wählen diesen Beruf häufig als Zweitausbildung und Wiederein-

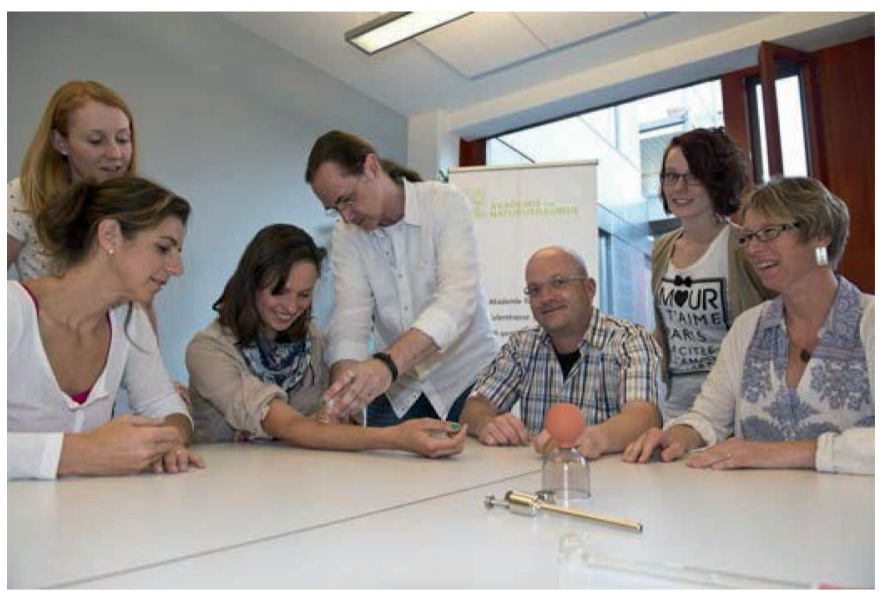

Abb. 3.

Praktischer Unterricht in der TEN. 
stieg. Frauen scheint die Naturheilkunde mehr anzusprechen. Vielleicht fühlen sie sich in einem Beruf, der Intuition, Sensibilität und Empathie braucht, am richtigen Ort. Und möglicherweise steht bei Frauen der materielle Aspekt weniger im Vordergrund bei der Wahl eines Berufes - finanziell ist dieser Beruf nicht sehr attraktiv.

Frauen achten wahrscheinlich (gesellschaftlich bedingt) auch weniger auf Status. Je prestigeträch- tiger die Tätigkeit (Komplementärtherapeut - Heilpraktiker - Arzt), desto mehr Männer sind zu finden.

Die Verteilung zeigt sich in den beiden Berufsbildern auch unterschiedlich: Sind es beim Beruf des Heilpraktikers ca. doppelt so viele Frauen wie Männer, zeigt sich bei den Komplementärtherapeuten ein wesentlich drastischeres Bild - mit rund fünfmal mehr weiblichen als männlichen Therapeuten.»

\section{Persönlichkeitsentwicklung}

Die Persönlichkeitsbildung ist für ANHK/SAKE ein absolutes Muss, da für Naturheilpraktiker/innen eine hohe Sozialkompetenz unabdingbar ist. Selbst- und Sozialkompetenz wird in der prozessorientierten Gesamtausbildung, die nicht modularisiert ist, entwickelt wie auch weiterentwickelt. 